\title{
Aspects cytologiques de quelques éléments du métabolisme chez un Sporotrichum
}

\author{
par M. THIBAUT, M. ANSEL et M. LARIVIERE
}

Laboratoire de Parasitologie et de Mycologie ( $\mathrm{P}^{\mathrm{r}} \mathrm{M}$. LARIVIÈRE), U.E.R. des Cordeliers, 15, rue de l'Ecole-de-Médecine, F. 75 - Paris (6 $\left.6^{\circ}\right)$

\begin{abstract}
Résumé
Sporotrichum schenckii (Hetkoen et Perkins, 1900), a été examiné par les techniques habituelles, en microscopie photonique et en microscopie électronique. L'ultrastructure des lipides et du glycogène dans les hyphes est rapportée. Le glycogène observé après différentes méthodes de fixation et après augmentation du contraste, se présente sous forme typique de particules $\alpha$ et $\beta$, libres dans le hyaloplasme ou inclues dans une \& vacuole». Les inclusions lipidiques se voient dans la plupart des hyphes.
\end{abstract}

\section{Summary}

Sporotrichum schenckii (Hetkoen \& Perkins, 1900), was examined by routine technics of light and electron microscopy. Special attention was paid to the ultrastructure of lipids and glycogen in filamentous hyphae. Glycogen observed after different methods of fixation and electron staining, normally appears in the typical form of $\alpha$ and $\beta$ particles free in the hyaloplasm or included in a «vacuole». Lipid inclusions were present in many of the hyphae.

Les travaux des cytologistes du début du siècle ont conduit à mettre en évidence les produits du métabolisme fungique : en particulier lipides et glycogène. Récemment, des recherches effectuées en microscopie électronique, ont montré que ces différents 
composés apparaissent plus ou moins structurés. La présente note a pour but de décrire l'ultrastructure du glycogène et des lipides dans les hyphes du Sporotrichum schenckii (Hetkoen et Perkins, 1900).

\section{Matériel et Méthodes}

Nos recherches ont été effectuées sur la forme mycélienne de ce Sporotrichum, cultivé sur milieu de Sabouraud ou sur milieu au moût de bière gélosé, à la température du laboratoire.

La localisation des produits dı métabolisme a été faite, en microscopie optiqae, sur des cultures sur lames. Le glycogène a été mis en évidence par l'iode et par la méthode de Bauer à l'acide chromique et au réactif de Schiff. Parallèlement, des préparations-témoins ont été traitées à la ptyaline. Les lipides ont été mis en évidence par le Soudan III, le noir Soudan B et le bleu B.Z.L. en solution alcoolique.

Pour la microscopie électronique, les extrémités distales des hyphes en culture ont été prélevées et fixées :

- soit dans le permanganate de potassium à $0,60 \%$ dans le tampon acétatevéronal de $\mathrm{pH} 7,4$ pendant deux heures;

- soit dans la glutaraldéhyde à $5 \%$ dans le tampon phosphate, de $\mathrm{pH} 7,4$ pendant deux heures et quinze heures. Ces deux fixations à la glutaraldéhyde étaient suivies d'une postfixation osmique à $2 \%$ dans le tampon acétatevéronal pendant une heure. Les différentes fixations ont été effectuées soit à la température du laboratoire, soit dans la glace fondante. Les pièces ont été ensuite lavées dans le tampon correspondant, puis déshydratées par l'alcool et l'oxyde de propylène.

Elles ont été inclues dans des résines à fonction époxy: soit l'araldite, soit l'épon 812 , puis polymérisées. Les coupes ont été pratiquées avec un ultramicrotome Sorvall Porter-Blum MT I, muni de couteaux de verre. Elles ont été recueillies sur des treillis recouverts d'une membrane de formwar, pour le matériel inclus dans l'araldite et sur des grilles à mailles fines, sans membrane support, pour celui inclus dans l'épon. L'examen et la photographie des coupes ont été effectués aux microscopes électroniques Hitachi modèle HU II B, Siemens Elmiskop I et Jem 100 U Jéolco.

\section{Résultats.}

\section{I. - En microscopie optique.}

La caractérisation cytochimique du glycogène se fait facilement. Il sa colore en brun par l'iode, en rouge par la méthode de Bauer. Dans les préparations-témoins traitées par la ptyaline, le glycogène disparaît.

Les lipides sont colorés en rouge par le Soudan III, en noir par le Soudan B, en bleu par le bleu B.Z.L. Nous pouvons voir alors, en suivant les hyphes en culture sur lame, depuis leur extrémité distale en voie d'allongement jusque vers leur point 
de départ proximal, que les lipides n'apparaissent que loin en arrière de l'extrémité distale : dans des hyphes mesurant de $1 \mu$ à $1,5 \mu$ de diamètre, c'est seulement de 30 à $50 \mu$ en arrière de ce front de croissance. L'apex des hyphes ne renferme pas de gouttelettes lipidiques; seules les portions adultes situées en arrière en présentent. Ceci déjà corrobore de façon visuelle les études physiologiques de Lindner, qui, mesurant les quantités de lipides produits, dans une masse donnée de mycélium, au cours d'une période de croissance, a montré que le matériel de réserve apparaît brusquement et seulement au bout d'un certain temps, alors que la croissance est déjà commencée.

\section{II. - En microscopie électronique.}

\section{A) Ultrastructure DU GLycogène.}

Sur les micrographies prises à un fort grandissement, le glycogène se présente sous forme de grains denses. On distingue deux types de particules à forte densité, que nous appellerons selon la nomenclature proposée par Drochmans :

- d'une part des éléments complexes en rosette, dont le diamètre le plus habituel est d'environ $150 \mathrm{~m} \mu$ : ce sont les particules $\alpha$ (fig. 1);

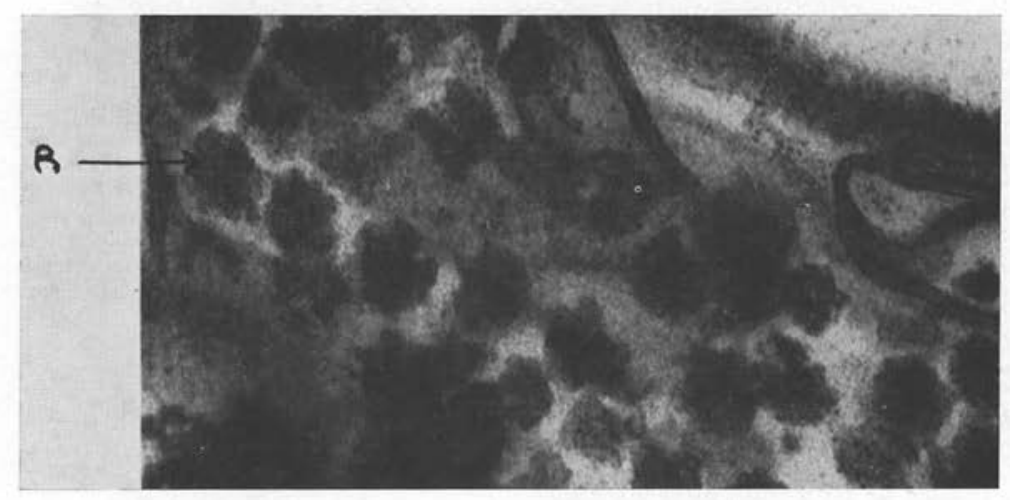

FIG. 1. - Coupe longitudinale d'une hyphe du Sporotrichum schenckii, montrant les rosettes de glycogène formées par la juxtaposition de particules $\beta$. $\times 110.000$. Fixation: glutaraldéhyde-tétroxyde d'osmium; contrastation: acétate d'uranylecitrate de plomb

R. : rosette de glycogène.

- d'autre part des particules $\beta$, de 15 à $30 \mathrm{~m} \mu$. de diamètre, dont la juxtaposition forme les rosettes. Mais les particules $\beta$ ne sont pas toujours associées, et peuvent rester plus ou moins dispersées dans les aires de glycogène, ce qui peut évoquer un phénomène de dissociation. Il faut aussi souligner la similitude d'aspect et de taille entre les particules $\beta$ et les ribosomes libres dans le hyaloplasme. Les grains de Palade peuvent en effet se grouper en amas, ressemblant aux particules $\beta$. 
Les plages de glycogène sont, soit libres dans le hyaloplasme de l'hyphe, soit séparées du hyaloplasme avoisinant par une membrane lisse de $15 \mathrm{mu}$ environ d'épaisseur (fig. 2). La membrane de ces « vacuoles à glycogène * a déjà été observée par Beaulaton (1964). Elle est bien préservée par la double fixation glutaraldéhyde et tétroxyde d'osmium.

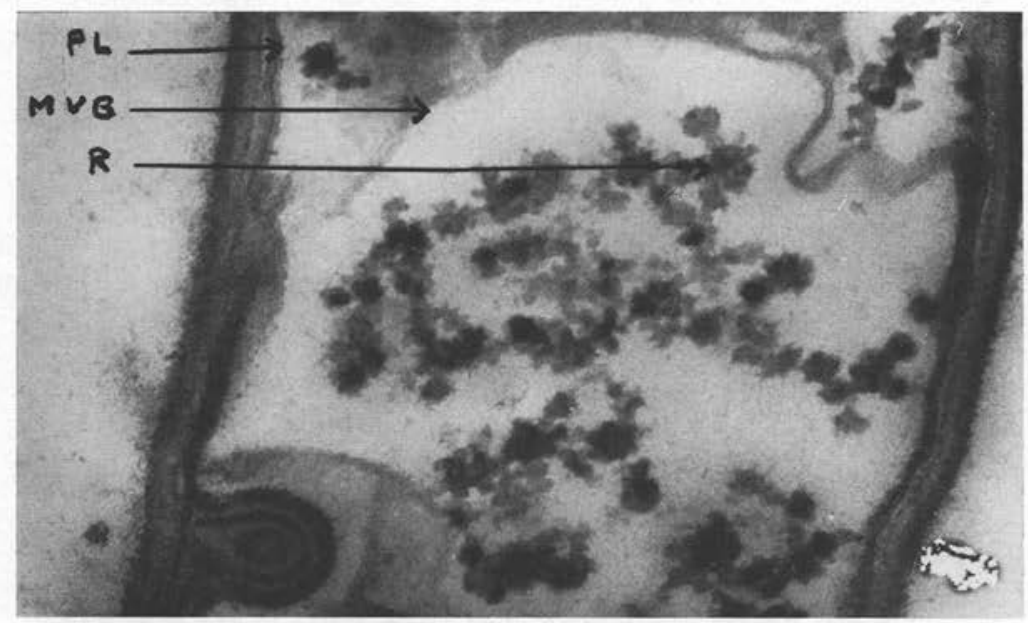

FIG. 2. - Coupe longitudinale d'un filament mycélien du Sporotrichum schenckii, montrant l'aspect d'un petit amas de glycogène. Les particules $\beta$ apparaissent irrégulièrement agglomérées en particules $\alpha$. Remarquer la présence d'une membrane séparant l'amas de glycogène du hyaloplasme environnant. $\times$ 54.000. Fixation: glutaraldéhyde-tétroxyde d'osmium; contrastation: acétate d'uranyle-citrate de plomb. PL: plasmalemme; MVG: membrane de la «vacuole à glycogène» R. : rosette de glycogène.

\section{B) Ultrastructure DES LIPIDES.}

Les lipides se font d'abord remarquer :

$1^{\circ}$ Par des plages claires, homogènes, arrondies ou ovoïdes, qui sont dues à leur dissolution par les alcools et l'oxyde de propylène (fig. 3), au cours des bains précédant l'inclusion dans l'épon ou l'araldite. Il est vraisemblable, comme l'ont suggéré divers auteurs, que ces lipides dissous ne présentent pas ou pas encore la constitution chimique de gouttelettes lipidiques, parfaitement mises en évidence, avec les mêmes procédés de fixation et de contrastation, malgré l'usage des alcools et de l'oxyde de propylène.

$2^{\circ}$ Les globules lipidiques que nous observons dans nos préparations ont été mis en évidence aussi bien après fixation au permanganate de potassium, qu'après fixation double à la glutaraldéhyde et au tétroxyde d'osmium. Nous avons toujours utilisé en plus la double contrastation à l'acétate d'uranyle et au citrate de plomb. Les globules 


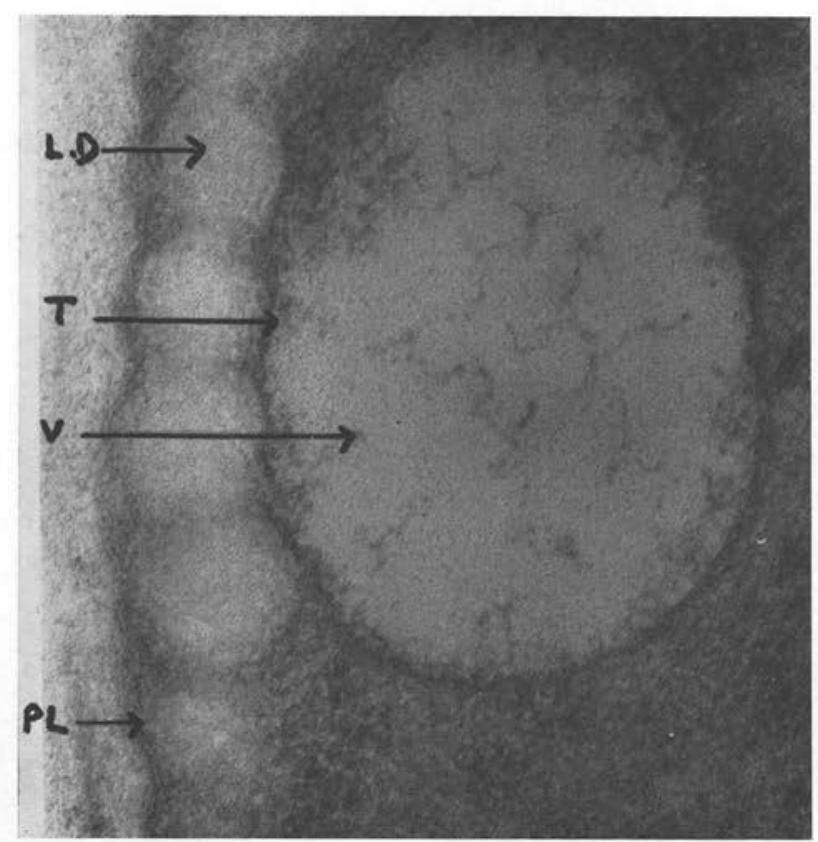

FIG. 3. - Coupe longitudinale d'une hyphe du Sporotrichum schenckii montrant l'aspect de lipides dissous. $\times$ 96.000. Fixation: glutaraldéhyde-tétroxyde d'osmium, contrastation: acétate d'uranyle-citrate de plomb. LD : lipides dissous ; T: tonoplaste ; $\mathrm{V}$ : vacuole; PL: Plasmalemme.

lipidiques se présentent sous une forme arrondie ou ovoïde (fig. 4). Leur surface esı godronnée, leur périphérie est bordée par une ligne dense aux électrons (fig. 5 et 6). Il ne nous semble pas qu'il faille voir dans cette ligne sombre une membrane, car on n'y observe jamais la structure tripartite caractéristique des membranes biologiques. On ne saurait donc l'assimiler ni au tonoplaste, ni au plasmalemme. Ces globules lipidiques sont situés quelquefois en dehors de la membrane plasmique (fig. 7), mais le plus souvent, ils se trouvent à l'intérieur même du hyaloplasme.

\section{Discussion.}

Nous n'avons jamais observé l'agglutination de globules lipidiques à l'intérieuı d'une sorte de vésicule, limitée par une membrane, formation qui a été décrite sous le terme de «sphérosomes », par Pihakaski chez deux espèces d'Hépatiques.

D'autre part, nous n'avons pas observé de dissolution d'un pigment caroténoïde, dans les zones où se trouvent normalement les globules lipidiques. Selon Schrantz, une telle dissolution se manifeste dans les paraphyses de certains Ascomycètes, à hyménium rouge, mais le Sporotrichum ici étudié n'est pas caroténogène. 
Les images de globules lipidiques, que nous avons obtenues après fixation à la glutaraldéhyde et au tétroxyde d'osmium, sont meilleures que celles que donne la fixation au permanganate (fig. 8). Avec ce dernier fixateur, en effet, si l'interface hyaloplasme-globule iipidique est souvent très nette, par contre le globule lui-même n'apparaît que comme une plage claire. Ces observations confirment l'opinion de Wells, déclarant

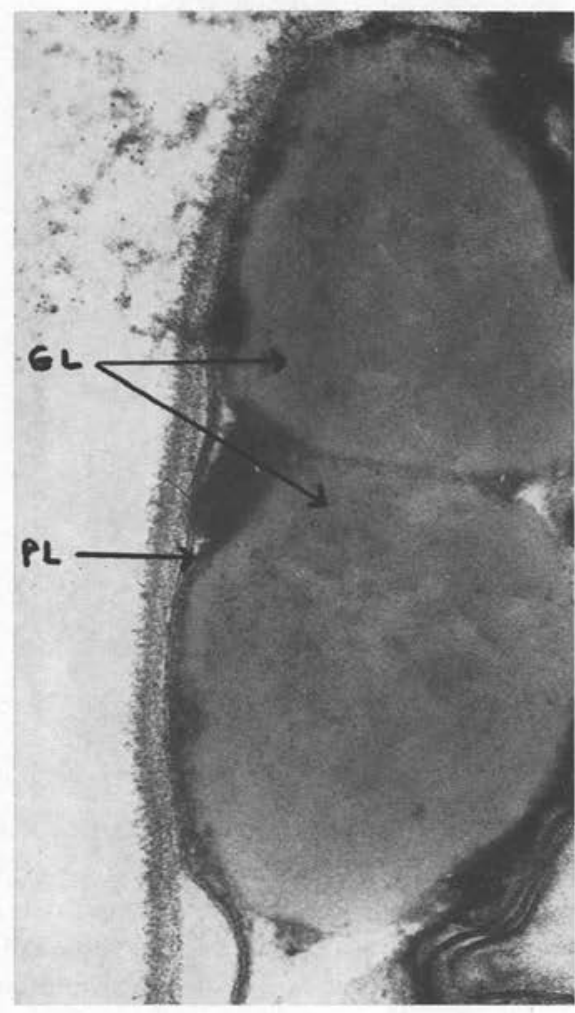

Fig. 4. - Coupe longitudinale d'une hyphe du Sporotrichum schenckii. On voit sur ce cliché deux globules lipidiques. $\times$ 67.500. Fixation : glutaraldéhyde-tétroxyde d'osmium ; contrastation: acétate d'uranyle-citrate de plomb. GL : globules lipidiques; PL: plasmalemme.

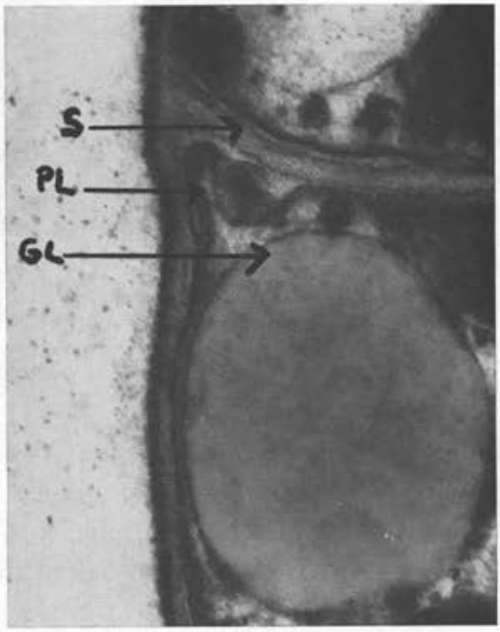

FIG. 5. - Coupe longitudinale d'un filament mycélien du Sporotrichum schenckii. Noter un globule lipidique bordé par une ligne dense aux électrons. $\times$ 36.000. Fixation: glutaraldéhyde-tétroxyde d'osmium; contrastation: acétate d'uranyle-citrate de plomb. S: septum; PL: plasmalemme ; GL: globule lipidique.

que seul le tétroxyde d'osmıum donne aux globules lipidiques une certaine densité aux électrons. Par contre, il est vraisemblable que si le tétroxyde d'osmium seul ne permet pas de voir la ligne dense périphérique, il dépend sans doute de la glutaraldéhyde qui lui est associée de donner des images nettes de cette surface. Cela conduit à se demander si la ligne qui la matériaiise existe réellement, la glutaraldéhyde la rendant plus évidente, ou si au contraire, elle n'est qu'un artéfact. 


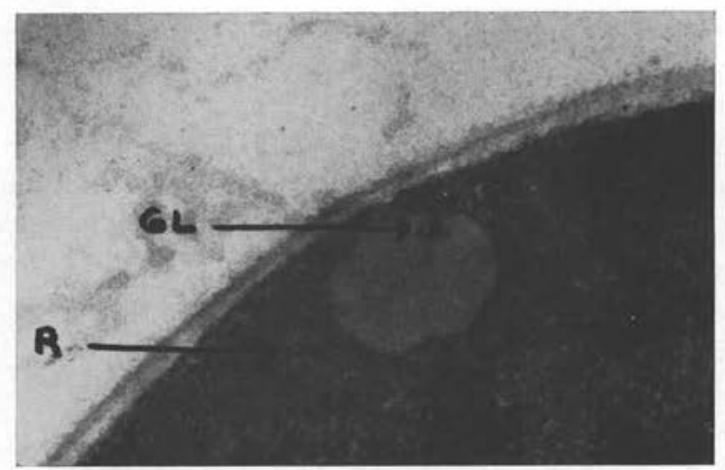

FIG. 6. - Globule lipidique dans une hyphe du Sporotrichum schenckii. $\times$ 45.000. Fixation: glutaraldéhydetétroxyde d'osmium; contrastation: acétate. d'uranylecitrate de plomb. GL: globule lipidique: R: ribosomes

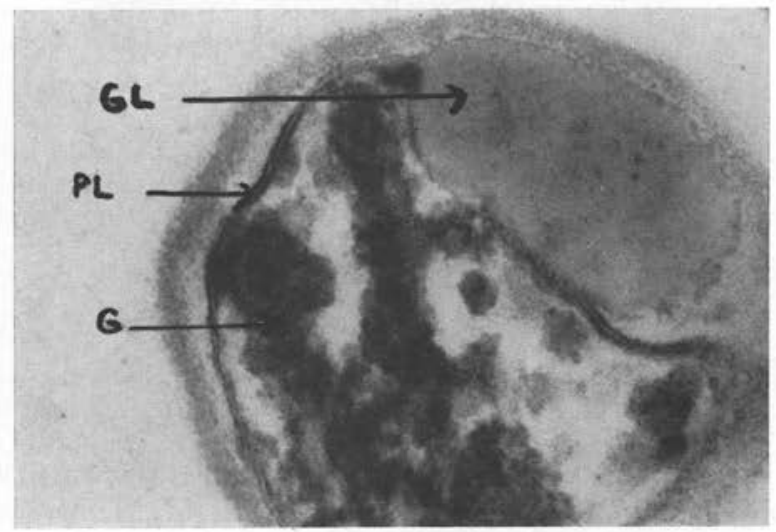

Fig. 7. - Coupe transversale d'un filament mycélien du Sporotrichum schenckii. Remarquer un globule lipidique situé en dehors du plasmalemme. $\times 67.500$. Fixation: glutaraldéhyde-tétroxyde d'osmium; contrastation: acétate d'uranyle-citrate de plomb. GL: globule lipidique; PL: plasmalemme; $G$ : glycogène

Le glycogène a été mis en évidence, en microscopie électronique, chez quelques champignons, par Zalokar (1961), Delay (1966), Scannerini (1967) et Schrantz (1968). L'aspect ultrastructural que nous avons observé est tout à fait conforme aux descriptions données par ces auteurs et semblable à celui qu'a défini Drochmans (1962) pour le glycogène du foie du rat. Mais Drochmans distingue en outre, des structures filamenteuses $\gamma$ de 3 mu. Ces particules $\gamma$ ne sont observées que lorsqu'on opère à un $\mathrm{pH}$ inférieur à 3. Nous ne les avons jamais mises en évidence chez le Sporotrichum schenckii, car nous avons toujours travaillé à un $\mathrm{pH}$ voisin de 7 . Schrantz n'a pas non 
plus reconnu ces structures, quel que soit le grandissement utilisé, ce qui n'a rien d'étonnant, lorsqu'on connaît les conditions précises de $\mathrm{pH}$ où l'on peut les observer.

Certains auteurs ont montré que le glycogène peut se présenter sous des aspects différents, en rapport avec le mode de fixation et de coloration, mais aussi avec le niilieu d'inclusion. Beaulaton (1968), travaillant sur la glande prothoracique de vers à soie, a obtenu le glycogène à l'état masqué après fixation glutaraldéhydique osmique, suivie d'une longue coloration à l'acétate d'uranyle. Schrantz (1968), après la même fixation, a obtenu, chez le Galactinia plebeia, des zones claires qui se situent aux empla-

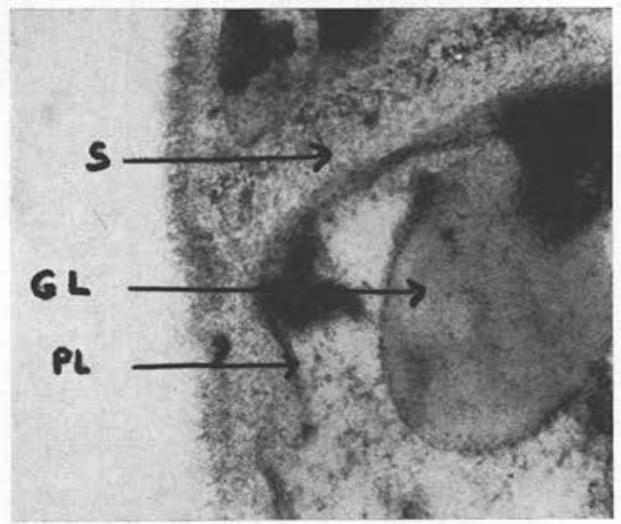

FIG. 8. - Coupe longitudinale d'une hyphe du Sporotrichum schenckii. Globule lipidique. $\times$ 75.000. Fixation: permanganate de potassium; contrastation: acétate d'uranyle-citrate de plomb. S: septum ; GL : globule lipidique ; PL: plasmalemme

cements où les autres techniques révèlent du glycogène. Drochmans (1962) affirme que le fixateur qui préserve le mieux le glycogène est le permanganate de potassium. Cependant, Fain-Maurel (1967) dans la glande hypobranchiale des Ptéropodes Thécosomes, après fixation par la glutaraldéhyde et le tétroxyde d'osmium, a obtenu l'aspect ultrastructural typique du glycogène. Revel, Napolitano et Fawcett (1961), puis Drochmans (1962) ont observé que les coupes de glycogène, fixées au permanganate et colorées à l'hydroxyde de plomb, montrent des particules bien isolées dans le cas de l'inclusion à l'araldite, tandis que, après inclusion au méthacrylate, les mêmes particules apparaissent réunies entre elles, d'une manière qui semble artificielle. Delay, dans une étude sur les asques de l'Ascobolus immersus (1966), fixant tantôt au permanganate de potassium, tantôt à la glutaraldéhyde-osmium, opérant soit à la température du laboratoire, soit à $+4^{\circ}$, a remarqué que la présence de glycogène dans ses microphotographies n'était pas constante et conclut que sa bonne conservation doit varier avec des facteurs qui restent à déterminer. Chez le Sporotrichum schenckii, nous avons toujours obtenu des images de glycogène : quel que soit le fixateur utilisé (permanganate ou glutaraldéhydetétroxyde d'osmium); quelle que soit la température de la fixation (température du 
laboratoire ou glace fondante); quel que soit le milieu d'inclusion (épon 812 ou araldite). Mais nous n'avons jamais fait d'inclusions dans le méthacrylate. Nous pensons que la bonne conservation du glycogène doit dépendre de facteurs autres que la nature des fixateurs ou la température de la fixation.

D'autre part, Beaulaton (1964) a montré que des particules morphologiquement identiques au glycogène peuvent s'accumuler dans certaines mitochondries des cellules sécrétrices d'Antheraea. Chez le Sporotrichum schenckii nous n'avons fait aucune observation mettant en évidence l'accumulation intramitochondriale de glycogène. La présence de glycogène dans ces organites supposerait d'ailleurs que les mitochondries puissent acquérir les systèmes enzymatiques de synthèse des polysaccharides. Comme cet auteur, nous avons mis en évidence des «vacuoles à glycogène » chez le Sporotrichum, mais nous n'avons jamais observé que ces « vacuoles » chargées de glycogène «possèdent des reliquats de structures mitochondriales ».

Carasso (1960) a signalé, chez des animaux, le rôle du réticulum endoplasmique dans la synthèse du glycogène. Fain-Maurel (1967) a émis l'hypothèse que l'ergastoplasme pourrait intervenir dans le métabolisme de ce corps. Dans les jeunes cellules de l'Algue rouge Lomentaria baileyana, Bouck a observé que de petits grains d'amidon floridéen sont fréquemment situés entre les citernes adjacentes du réticulum endoplasmique. Dans les hyphes du Sporotrichum schenckii, nous n'avons jamais constaté jusqu'à ce jour que le glycogène soit associé aux membranes de l'ergastoplasme. D'autre part, si le glycogène et l'amidon floridéen se colorent également en brun acajou par l'iode, l'ultrastructure de ces deux composés est très différente.

$\mathrm{Si}$ la microscopie photonique ne donne qu'une détection approximative du glycogène et des lipides, dans des filaments aussi ténus que ceux du Sporotrichum schenckii, la microscopie électronique permet non seulement d'en certifier la présence, mais encore d'en décrire l'aspect morphologique et l'infrastructure. Le glycogène et les globules lipidiques apparaissent comme des substances de réserve, dont la genèse s'accomplit, après une phase de croissance, dans les portions plus âgées et plus mûres des filaments. Ce glycogène se révèle fondamentalement de même structure que celui décrit chez les animaux par Drochmans, mais très différent de l'amidon floridéen.

\section{Bibliographie}

Beaulaton (J.), 1968. - Modifications ultrastructurales des cellules sécrétrices de la glande prothoracique de vers à soie au cours des deux derniers âges larvaires. II. Le glycogène, ses relations avec le chondriome et le réticulum endoplasmique. J. Microsc., 7, 673-692.

—, 1962. — La morphologie du glycogène dans la glande prothoracique de Philosamia cynthia Drury, au cours de la diapause nymphale. J. Microsc., 1, 469-472.

-, 1964. - Sur l'accumulation intramitochondriale de glycogène dans la glande prothoracique du ver à soie du Chêne Antheraea pernyi (Guér) pendant les quatrième et cinquièmes stades larvaires. C.R. Acad. Sci., 258, 4139-4141. 
BoucK (G.-B.), 1962. - Chromatophore development, pits and other fine structure in the red alga Lomentaria baileyana (Harv.), Farlow, J. Cell. Biol., 12, 553-569.

Bracker (C. E), 1967. - Ultrastructure of fungi. Ann. Rev. Phytopathol., 5, 343-374.

Carasso (N.), 1960. - Rôle de l'ergastoplasme dans l'élaboration du glycogène au cours de la formation du paraboloïde des cellules visuelles. C.R. Acad. Sci., 250, 600-602.

Delay (C.), 1966. - Etude de l'infrastructure de l'asque d'Ascobolus immersus Pers. pendant la maturation des spores. Ann. Sc. nat., 7, 361-378.

Drochmans (P.), 1960. - Mise en évidence du glycogène dans la cellule hépatique par microscopie électronique. J. Biophys. Biochem. Cytol., 8, 553-558.

—, 1962. - Morphologie du glycogène. Etude au microscope électronique de colorations négatives du glycogène particulaire. J. Ultrastruct. Res., 6, 141-163.

EHRLICH (H. G.) et EHRLICH (M. A.), 1966. - Ultrastructure of the hyphae and haustoria of Phytophtora infestans and hyphae of Phytophtora parasitica. Can. J. Bot., 44, 1495-1503.

ERrera (L.), 1906. - L'épiplasme des Ascomycètes et le glycogène des végétaux. Recueil de l'Inst. Bot. (Univ. de Bruxelles), 1, 1-68.

FAIN-MAUREL (M.-A.), 1966. - Localisations intramitochondriales et intracisternale du glycogène monoparticulaire. C.R. Acad. Sci., 263, 1107-1110.

-, 1967. - Caractères infrastructuraux de la glande hypobranchiale des Ptéropodes Thécosomes. J. Microsc., 6, 599-616.

-, 1967. - Nouvelles observations sur la localisation du glycogène dans l'ergastoplasme. C.R. Acad. Sci., 265, 126-129.

FAWCETT (D. W.), 1966. - An atlas of fine structure: the cell, its organelles and inclusions, Saunders, Philadelphia, 448 p.

Guilliermond (A.), 1903. - Contributions à l'étude de l'épiplasme des Ascomycètes et recherches sur les corpuscules métachromatiques des Champignons. Ann. Myc., 1, 201-215.

—, Mangenot (G.) et Plantefol (L.), 1933. - Traité de cytologie végétale, Le François éd., Paris.

HAWKer (L. E.) et Aввot (P. Mc. V.), 1963. - Fine structure of vegetative hyphae of Rhizopus. J. Gen. Microbiol., 30, 401-408.

-, 1965. - Fine structure of Fungi as revealed by electron microscopy. Biol. Rev., 40, $50-92$.

JenSEN (W. A.), 1962. - Botanical histochemistry, Freeman et Co., San Francisco, 408 p.

PIHAKasKI (K.), 1966. - An electron miscroscope study in the oil bodies of two hepatic species. Protoplasma, 4, 393-399.

ReVel (J. P.), 1964. - Electron microscopy of glycogen. J. Histochem. Cytochem, 12, 104-114.

-, Napolitano (L.) et Fawcett (D. W.), 1960. - Identification of glycogen in electron micrographs of thin tissue sections. J. Biophys. Biochem. Cytol., 8, 575-589.

SCANNERINi (S.), 1967. - Un particolare sistema di membrane e lomasomi in Agaricus campester, Fr. var. bisporus. Caryologia, 20, 115-133. 
Schrantz (J.-P.), 1964. - Etude au microscope électronique des synapses de deux Discomycètes: Peziza aurantia Pers. ex. Fr. et Ciliaria hirta Schum Boudier. C.R. Acad. Sci., 258, 3342-3344.

—, 1968. - Ultrastructure et localisation du glycogène chez l'Ascomycète Galactinia plebeia Le Gal. Rev. Cytol. et Biol. Vég., 31, 151-154.

Wells (K.), 1964. - The basidia of Exidia nucleata. I. Ultrastructure. Mycologia, 56, 327-341.

-, 1965. - Ultrastructural features of developing and mature basidia and basidiospores of Schizophyllum commune. Mycologia, 57, 236-261. 